\title{
Determining the Pressure Gain of Pressure Gain Combustion
}

\author{
Thomas A. Kaemming, ${ }^{1}$ \\ Innovative Scientific Solutions, Inc., Dayton, Ohio, 45459 \\ Daniel E. Paxson ${ }^{2}$ \\ NASA Glenn Research Center, Cleveland, Ohio, 44130
}

\begin{abstract}
Over the past few decades, there has been significant research into propulsion concepts attempting to employ pressure gain combustion. Pressure gain combustion concepts to date have resulted in dynamic, non-uniform gas flows which are difficult to characterize and compare with more conventional forms of propulsion. This paper proposes a technique to derive for the pressure gain combustion device an equivalent, steady, uniform gas pressure that is available to do work or provide thrust, thereby providing a direct comparison with conventional propulsive devices.
\end{abstract}

\section{Nomenclature}

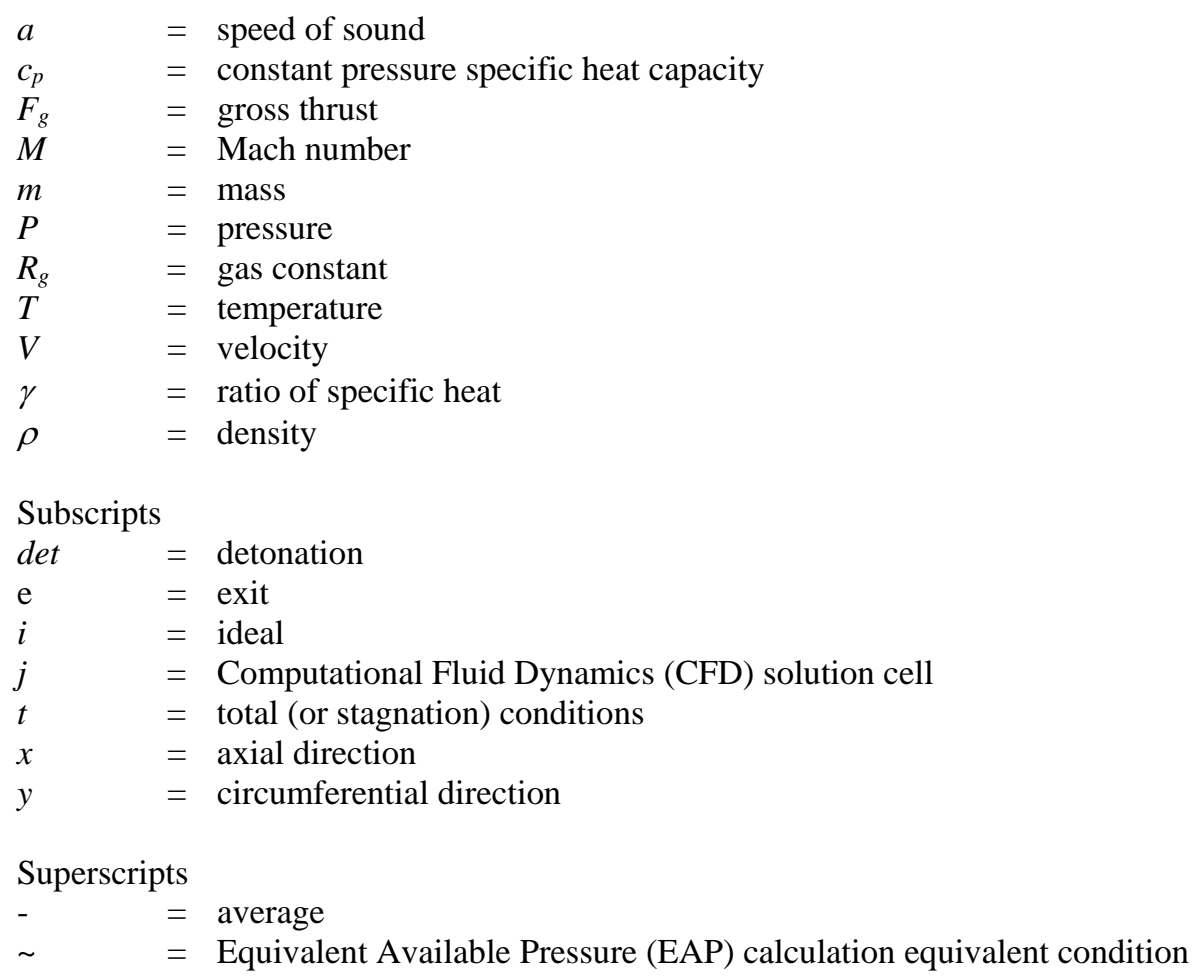

Analytical Stations

$0=$ ambient

$3=$ combustor entrance

\footnotetext{
${ }^{1}$ Research Engineer, 7610 McEwen Rd., AIAA Associate Fellow

2 Aerospace Research Engineer, Research and Engineering Directorate, 21000 Brookpark Road/MS 77-1, AIAA Associate Fellow
} 


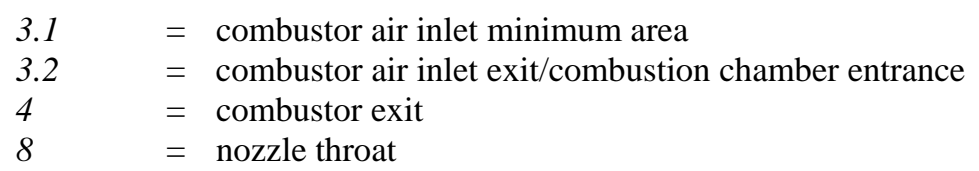

\section{Introduction}

$\mathrm{O}$ ver the last several years, there has been a resurgence of interest in pressure gain combustion (PGC) using many implementation approaches, including pulsed detonation engines (PDE), rotating detonation engines (RDE), and constant volume combustion systems, such as wave rotors, as well as others. While different in their implementations, all of these devices promise improved thermodynamic performance as a result of their increased combustion pressures. However, all of these PGC devices are unsteady and, as such, a reasonable and conclusive validation of their promised pressure gain is elusive. The harsh, unsteady combustor environment precludes the use of any state-of-the-art in-situ total pressure measurements. Furthermore, it is not obvious that a simple average of exit total pressure is a meaningful measure of available total pressure. Therefore, an acceptable means of deducing pressure gain from measureable quantities is needed.

This paper examines PGC computational results that serve as the basis for a proposed means of deducing the pressure gain. A consistent means of computing the pressure gain is provided for general PGC computational results. Sample results of reduced computational fluid dynamic (CFD) properties are provided. In addition, a consistent means of estimating pressure gain specifically for the RDE is proposed using experimentally measureable quantities. The proposed experimental procedure is exercised using CFD results as a surrogate for experimental results to demonstrate its effectiveness.

\section{Challenges}

To illustrate the challenges associated with determining the pressure gain in a PGC device, an example of CFD computed RDE combustor temperature, pressure and axial Mach number contours is provided in Fig. 1. This example, and all others to follow utilize a stoichiometric hydrogen/air case with a feed plenum pressure of 4 atmospheres and an exit pressure of 1 atmosphere. The feed plenum temperature is $540^{\circ} \mathrm{R}$. The CFD depiction is in the detonation frame-of-reference and shows the RDE annular combustor unwrapped for a 2-D representation. For this paper, ' $\mathrm{x}$ ' direction is axial and ' $y$ ' direction is circumferential. Both dimensions are non-dimensionalized (normalized) by the RDE circumference.

Examining the exit plane at the top of Fig. 1, one can observe that the exit static pressure varies roughly 3:1 in magnitude as the detonation circumnavigates the annular combustor at a pace greater than 1,000 times per second. In addition, the exit plane temperatures can approach values an order-of-magnitude greater than the inflow temperature. The combination of these high temperatures and high frequency of the operation make direct measurement of the flow's stagnation pressure impractical with currently available instrumentation. Current RDE testing typically includes measurement of average wall static pressures using Capillary Tube Averaged Pressure (CTAP) [1] and somewhat muted dynamic wall static pressures using Infinite Tube Pressure (ITP) sensors.

\section{A Representative Pressure}

The community must first agree upon the definition of the pressure available in pressure gain combustion. Based on prior work [2], the authors propose the use of Equivalent Available Pressure (EAP). The definition of EAP is the "flow stagnation pressure which is representative of flow's ability to do work or provide thrust." Because the objective of PGC is to either directly provide thrust via an exhaust nozzle or do work through a turbine, EAP is the pressure required to produce the thrust or work provided by the PGC assuming that the flow is steady and homogeneous. In this way, EAP is directly comparable to uniform flow of a conventional combustor assuming the same mass flow and the same heat addition.

There are two practical EAP values available from any numerical PGC solution or experiment: 1) the EAP equivalent to the actual measured thrust or work obtained [3] and 2) the EAP equivalent to the thrust or work possible if the flow were expanded to ambient pressure. The former includes the performance of the downstream component, either nozzle or turbine, and therefore does not solely represent the combustor performance. The latter divorces the EAP from the downstream component performance and therefore represents the combustor performance alone. It is suggested that the latter be designated EAPi. EAPi is analogous to the ideal exit velocity in thrust calculations and ideal spouting velocity used in turbine performance calculations.

Pressure gain must be measured relative to a consistently defined pressure. The germane SAE (Society of Automotive Engineers) and JANNAF (Joint Army, Navy, NASA and Air Force) committees have provided 

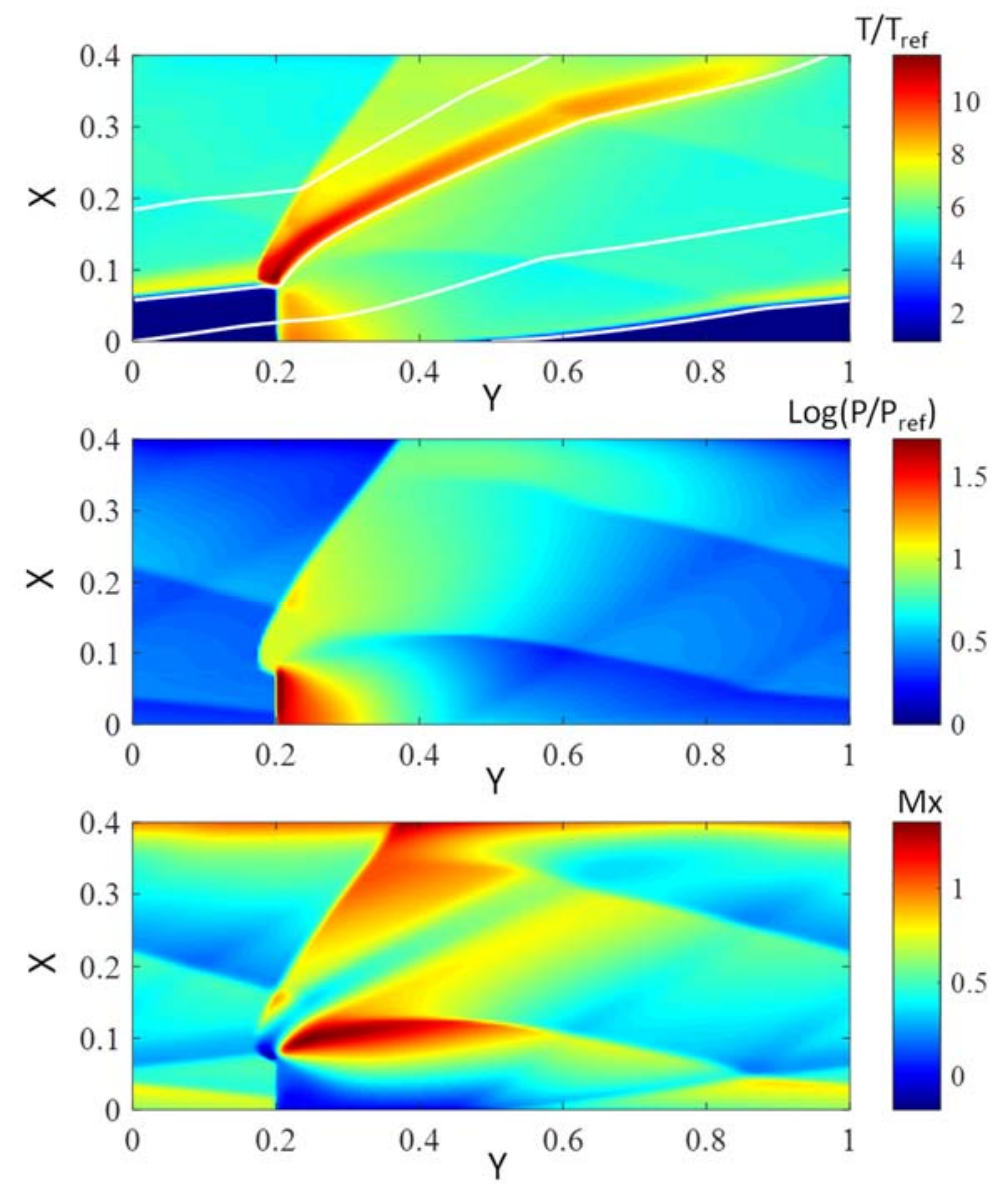

Fig. 1 Example Flow Conditions within a Rotating Detonation Engine

guidance that the PGC combustor must include all hardware specific to the PGC combustor. Therefore, any air inlet or air valving should be considered part of the PGC combustor. Under that guidance, the upstream pressure, used for the pressure gain definition, is the air being supplied to the combustor inlet, $P_{t 3}$.

To function properly, the combustor flow balance must be dictated by the downstream component, such as a nozzle or a turbine. This paper focuses on a ram RDE device, so the downstream component is a nozzle. To properly establish the flow balance, calculations in this paper will consider flow through the nozzle minimum area, $A_{8}$.

\section{Ideal EAP Based on Computational Results}

The PGC flow solutions provided by CFD provide valuable insight into the PGC operation. Because all of the essential flow properties are available, these CFD solutions can be used to directly compute an EAPi from the computer combustor exit conditions. In this paper, we take the following approach:

To compute the EAPi, we will:

a) compute the ideal stream thrust for each flow segment

b) combine all of the flow segments to stream thrusts to obtain a total flow ideal stream thrust

c) compute an EAPi which would provide the same total ideal stream thrust given a uniform flow.

To compute each flow segment's ideal stream thrust, we start by expanding each exit-plane flow segment isentropically to ambient pressure, $P_{0}$. In this example, we use the CFD analysis assumption of a thermally and calorically perfect gas. First, we compute the ideal exit Mach from the segment's total pressure by inverse solution of the isentropic flow function:

$$
\frac{\mathrm{P}_{8 \mathrm{t}, \mathrm{j}}}{\mathrm{P}_{0}}=\left[1+\frac{\gamma-1}{2} \mathrm{M}_{\mathrm{ei}, \mathrm{j}}^{2}\right]^{\gamma /(\gamma-1)}
$$


Where: $\mathrm{e}=$ exit; $\mathrm{i}$ = ideal; $\mathrm{j}$ = CFD exit plane solution point.

The ideal exit velocity is then computed from:

$$
\begin{gathered}
\mathrm{T}_{\mathrm{ei}, \mathrm{j}}=\mathrm{T}_{8 \mathrm{t}, \mathrm{j}} *\left[1+\frac{\gamma-2}{2} \mathrm{M}_{\mathrm{ei}, \mathrm{j}}^{2}\right] \\
\mathrm{V}_{\mathrm{ei,j}}=\mathrm{M}_{\mathrm{ei}, \mathrm{j}} * \mathrm{a}_{\mathrm{ei}, \mathrm{j}}=\mathrm{M}_{\mathrm{ei}, \mathrm{j}} * \sqrt{\gamma g \mathrm{R}_{\mathrm{g}} \mathrm{T}_{\mathrm{ei}, \mathrm{j}}}
\end{gathered}
$$

The total velocity must be resolved into its axial component to derive axial force and conserve non-axial energy:

$$
V_{x e i, j}=\sqrt{V_{e i, j}^{2}-V_{8 y, j}^{2}}
$$

The mass flux averaged axial ideal exit velocity is then computed. This velocity is directly related to the ideal specific thrust of the device and corresponds to the ideal spouting velocity.

$$
\bar{V}_{x e i}=\frac{\sum_{j=1}^{n} \rho_{j} A_{x, j} V_{x, j} V_{x e i, j}}{\sum_{j=1}^{n} \rho_{j} A_{x, j} V_{x, j}}
$$

The mass flux averaged total temperature, which represents the total energy for the EAPi calculation, is computed from:

$$
\bar{T}_{8 t}=\frac{\sum_{j=1}^{n} \rho_{8 j} A_{8 x, j} V_{8 x j} T_{8 t j}}{\sum_{j=1}^{n} \rho_{8 j} A_{8 x, j} V_{8 x j}}
$$

The EAPi must represent the total pressure which will provide the equivalent axial exit velocity for the same average total temperature. Therefore, the exit static temperature for the equivalent (i.e. EAP) flow must conserve total temperature (including non-axial energy) while expanding axially to the ideal exit velocity, $\bar{V}_{x e i}$. The EAP-related static temperature is computed by subtracting the axial velocity kinetic energy from the average total temperature:

$$
\tilde{T}_{e i}=\bar{T}_{8 t}-\frac{1}{2} \frac{\bar{V}_{x e i}^{2}}{c_{P}}
$$

With the average properties of the unsteady flow characterized, the equivalent EAPi that provides the mass flux averaged ideal axial exit velocity when expanded isentropically to $\mathrm{P}_{0}$ can be calculated:

$$
E A P_{i}=\tilde{P}_{8 t i}=P_{0} *\left[\frac{\bar{T}_{8 t}}{\tilde{T}_{e i}}\right]^{\frac{\gamma}{\gamma-1}}
$$

\section{A. Computational Methods}

For this paper, the analytical RDE results were obtained using a quasi-two-dimensional RDE CFD code that has been detailed in the literature [4-8]. The model basis is a high resolution, computational fluid dynamic (CFD) algorithm that integrates the quasi-two-dimensional, single-species, reactive Euler equations with source terms. The code adopts the detonation frame of reference and deliberately utilizes a course grid (i.e. is diffusive) in order to eliminate the highest frequency unsteadiness (e.g. detonation cells, Kelvin-Helmholtz phenomena). All of the results shown in this paper utilize a 200 points by 80 points uniformly spaced grid. The result is a flowfield solution that is invariant with time when converged. The working fluid is assumed to be a single, calorically perfect, premixed gas. Relevant properties used in all calculations of this paper are a real gas constant, $R_{g}$, of $73.92 \mathrm{ft}-\mathrm{lb}_{\mathrm{f}} / \mathrm{lb}_{\mathrm{m}} / \mathrm{R}$, and a ratio of specific heats, $\gamma$, of 1.264 [9-10].

The source terms contain sub-models which govern the reaction rate, momentum losses due to skin-friction, and the effects of heat transfer to the walls. The sub-models are adapted from validated one-dimensional sub-models used 
to investigate pulse detonation engines and other gasdynamic devices [11-13]. For the present idealized study, only the reaction rate sub-model will be used. Due to the simplicity of this sub-model, two reaction rate constants are required: a large one for regions of detonation and a smaller one for regions of deflagration. Based on past validation efforts with experiments $[10,14]$, the deflagrative rate constant is set to a value such that, in combination with other fluid dynamic processes, approximately $6 \%$ of the premixed RDE throughflow reacts in this region. The remaining $94 \%$ detonates.

The governing equations are integrated numerically in time using an explicit, second-order, two-step, Runge-Kutta technique. Spatial flux derivatives are approximated as flux differences, with the fluxes at the discrete cell faces evaluated using Roe's approximate Riemann solver. Second-order spatial accuracy (away from discontinuities) is obtained using a so-called MUSCL technique (i.e. Monotonic Upstream-Centered Scheme for Conservation Laws) whereby the primitive variable states within the cells are represented as piecewise linear rather than constant as first order accuracy would imply. Oscillatory behavior is avoided by limiting the linear slopes.

Considering an RDE where the circumferential direction is $\mathrm{y}$, and the axial direction is $\mathrm{x}$, the following boundary conditions are imposed. At $y=0.0$ and $y=1.0$, periodic (a.k.a. symmetric) conditions are used. These ensure that the $\mathrm{y}$-dimension of the computational space faithfully represents an annulus (which is continuous and has no boundary). At $x=x_{\max }$, constant pressure outflow is imposed along with characteristic equations to obtain $\rho$ and $V_{x}$ for the image cells. If the resulting flow is sonic, or supersonic, then the imposed pressure is disregarded. If, in addition, the upstream flow is supersonic, then $P, \rho$ and $V_{x}$ are extrapolated from the interior [15]. The possibility for a normal shock solution whereby supersonic outflow jumps to subsonic is also accommodated. The y-velocity component, $V_{y}$, is extrapolated from the interior at each boundary location. At $x=0.0$ (the inflow face), partially open boundary conditions are applied as described and validated in Ref. 16. This face is presumably fed by a large manifold at a fixed total pressure and temperature. The manifold terminates at the face and is separated from it via an orifice. The ratio of orifice flow area to RDE annulus area, $A_{3.1} / A_{3.2}$, is generally less than 1 . If the interior pressure, $P_{3.2}$, is less than the manifold pressure, $P_{t 3}$, then inflow occurs. The boundary condition routine determines $P, \rho$ and $V_{x}$ for the inflow face image cells subject to a momentum (total pressure) loss model which depends on the mass flow rate and the value of $A_{3.1} / A_{3.2}$. The routine is capable of accommodating a scenario where the inflow becomes choked. If $A_{3.1} / A_{3.2}$ is assigned a value of 1 , a lossless inlet is ensured. The y-velocity component, $V_{y}$, is prescribed during inflow, and it is here that a reference frame change is implemented. Rather than specify $V_{y}=0$ (i.e. no swirl) which is the laboratory or fixed frame condition, the negative of the detonation speed, $V_{\text {det }}$ is prescribed instead. As a result of this change to the detonation reference frame, the computational space becomes one where a steady-state solution is possible. If the interior pressure along the inlet face is greater than $P_{t 3}$, as might be found just behind the detonation, then there will be backflow into the manifold through the orifice. The boundary condition routine can accommodate this as well. However, for this investigation a notional check-valve boundary condition is implemented which detects when backflow would normally occur, and applies a solid wall boundary condition.

\section{B. Example Calculations}

As an example of EAPi calculation, the CFD solution presented in Fig. 1 will be examined. The RDE configuration analyzed has an air inlet minimum area, $A_{3.1}$, that is $60 \%$ of the combustion chamber flow area, $A_{3.2}$. At the combustor exit, there is a contracting nozzle with a minimum flow area, $A_{8}$, that is $80 \%$ of the combustion chamber flow area, $A_{3.2}$. The nozzle contraction is implemented as a smooth, continuous area profile over the last $15 \%$ of the axial length.

Some of the important flow parameters for this solution are presented in Table 1. The average (Avg) is a simple area weighted average (equivalent to a time-average in this frame of reference) and $P_{t 3.2} / P_{t 3}$ is the pressure recovery of the inflow (i.e. does not include detonation pressure rise). For this example, the detonation velocity was 6,000 $\mathrm{ft} / \mathrm{sec}(1,829 \mathrm{~m} / \mathrm{sec})$.

The inlet pressure recovery, $P_{t 3.2} / P_{t 3}$, illustrates the wide dynamics the inlet undergoes during RDE operation. As the pressure ratio across the inlet varies through a cycle, the pressure recovery across the inlet also varies. Of particular note is the significant variation in the flow's major exit properties, where total pressure varies over 200\%. The objective is to compute a representative total pressure for an equivalent uniform steady flow.

The mass flux averaged ideal axial exit velocity for this example is $5,157 \mathrm{ft} / \mathrm{sec}(1,572 \mathrm{~m} / \mathrm{sec})$. The corresponding mass flux averaged total temperature is 3,744 deg $\mathrm{R}(2,080 \mathrm{deg} \mathrm{K})$. The equivalent static temperature for the mass flux averaged total temperature and computed ideal axial exit velocity is 2,577 deg $\mathrm{R}$ (1,432 deg K). The resultant EAPi is 87.9 psia $(6.0 \mathrm{~atm})$. This EAPi represents a $49 \%$ pressure gain across the combustor when compared to the air feed plenum pressure. 
Table 1 Example CFD Flow Parameters

\begin{tabular}{|c|c|c|c|c|}
\hline & Max & Min & Avg & $\begin{array}{c}\text { Max- } \\
\text { Min)/Avg }\end{array}$ \\
\hline $\mathrm{P}_{\mathrm{t} 3.2} / \mathrm{P}_{\mathrm{t} 3}$ & 0.85 & 0.64 & 0.74 & $29 \%$ \\
\hline $\mathrm{P}_{\mathrm{t} 8} / \mathrm{P}_{\mathrm{t} 3}$ & 4.07 & 0.67 & 1.43 & $237 \%$ \\
\hline $\mathrm{T}_{\mathrm{t} 8} / \mathrm{T}_{\mathrm{t} 3}$ & 8.07 & 5.37 & 6.57 & $41 \%$ \\
\hline $\mathrm{M}_{8 \mathrm{x}}$ & 1.33 & 0.86 & 0.99 & $48 \%$ \\
\hline
\end{tabular}

To put these results into a visual perspective, the total pressure for each computational cell at three major engine stations are provided in Fig. 2. The three stations are: 3 air feed plenum, 3.2 entrance of the combustor and 4 exit of the combustor. To provide further insight, the total pressures at the entrance to the combustor, station 3.2, are broken down into the pre-combustion, unburned gases and the post-combustion, burned gases.

The figure illustrates the vast spread of the total pressures through the RDE. From the feed plenum to the unburned gases, there is roughly an average total pressure loss of $26 \%$. The detonation then raises the gas total pressure anywhere between the unburned gas total pressure up to 93 atmospheres at the peak of the spike. As the combusted gases expand to the combustor exit, the total pressures drop until the total pressure spread is now from roughly the unburned gas total pressures up to 16 atmospheres.

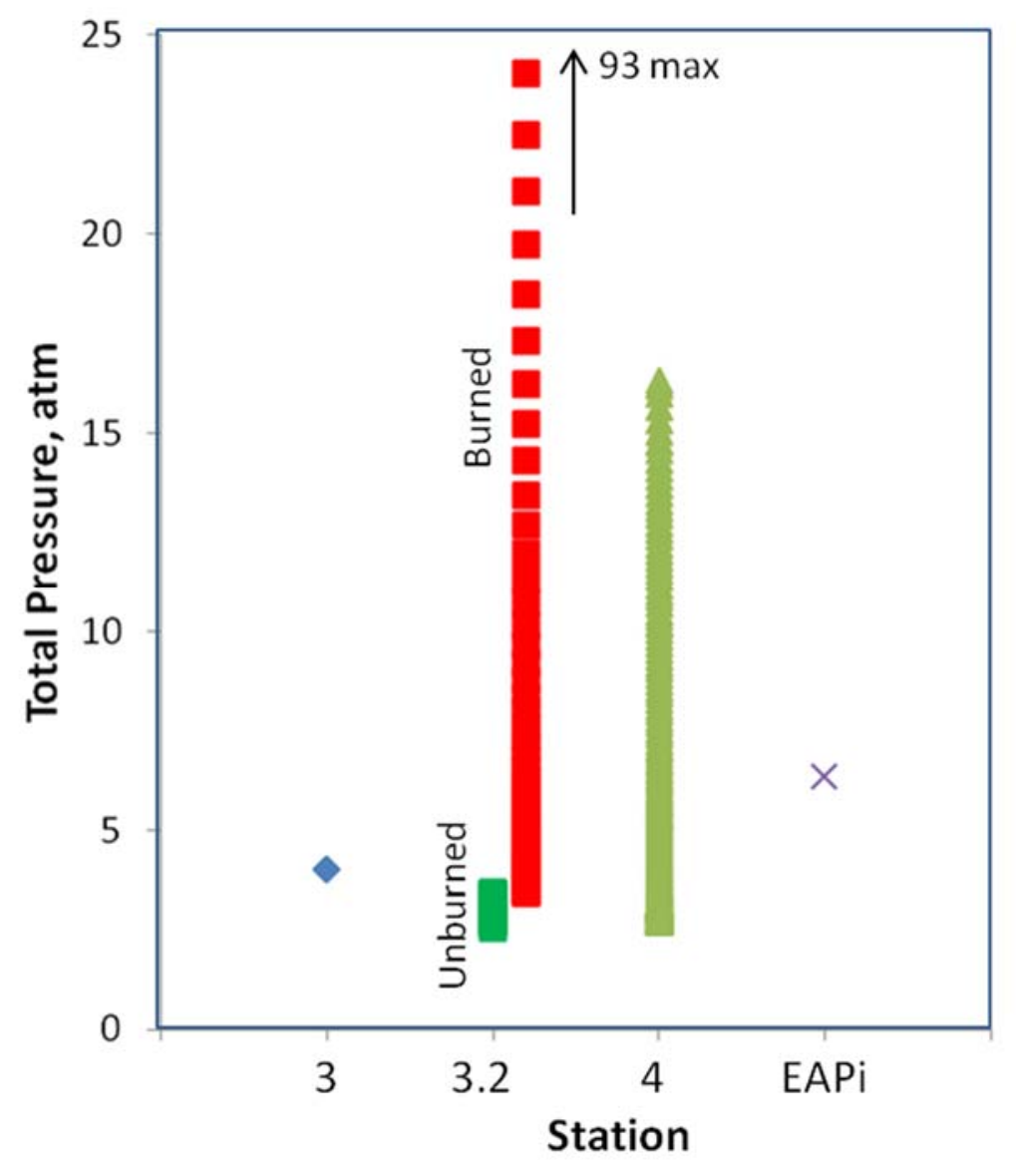

Fig. 2 Example CFD Predicted Total Pressures with RDE Combustor 
The large dispersions of the gas total pressures create the challenge of selecting a single total pressure value to quote for the combustor pressure gain. The rationale of this paper is to compute an EAPi which represents a uniform flow that provides the same ideal thrust as the integrated ideal thrust of all streams.

\section{Non-Axial Momentum}

For RDE combustors, the circumferential movement of the detonation wave induces some non-axial flow. While for most cases there is no net momentum associated with this non-axial momentum, there is some energy that is not readily available for axial thrust or for turbine work. Because this momentum is not easily recovered, the proposed definition of EAPi provided here assumes that the non-axial energy is not available. Further study is necessary to assess the effect of the non-axial momentum on available turbine work.

To illustrate the magnitude of this effect, the baseline CFD solution was analyzed assuming that the non-axial energy is not available (as described above, Eq. (7)) and then as if it was available. To include the non-axial energy into the computed EAP, the calculation of the static temperature at exit ideal conditions includes the average total exit velocity:

$$
\tilde{T}_{e i}=\bar{T}_{t}-\frac{1}{2} \frac{\bar{V}_{e i}^{2}}{c_{P}}
$$

The new EAPi can then be computed as before. For the baseline CFD of this report, the inclusion of the non-axial energy in the EAPi calculation resulted in a 6\% increase in EAPi.

Means of minimizing the non-axial momentum have been postulated, such as increased combustor length and/or mixing of the combustor exhaust [17]. However, none of these techniques has yet been proven to be effective. In addition, the overall usefulness of these geometries, when the increased length, weight, heat transfer, etc., are considered, is questionable. If such geometries are studied, the EAPi calculation provided here will still be representative because their geometry will have converted the non-axial momentum to axial momentum prior to the nozzle throat.

\section{RDE Geometry Effects}

To illustrate the sensitivity of EAPi to the primary RDE combustor geometries, the RDE inlet area and nozzle throat area were varied in the CFD. The resulting CFD data were reduced as described above. The resultant EAPi, expressed as "Pressure Gain”, is presented in Fig. 3.

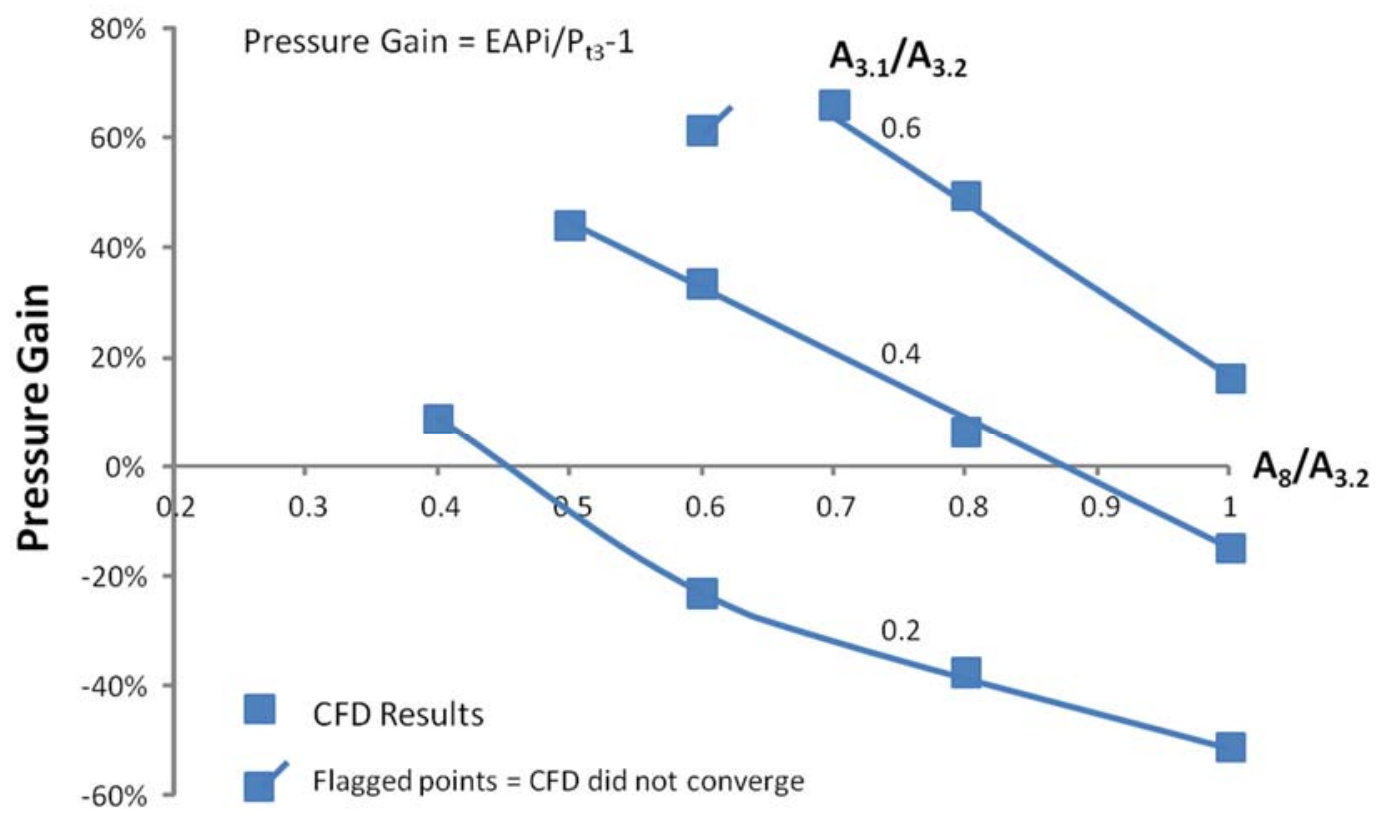

Fig. 3 Combustor Pressure Gain Computed from CFD 
The pressure gain of Fig. 3 shows an intimate relationship between the RDE air inlet minimum area, $A_{3.1}$, and the RDE nozzle minimum area, $A_{8}$. Small inlet areas compared to nozzle areas result in the inlet being choked over much of the RDE cycle. This choking results in significant total pressure losses across the inlet, which is detrimental to EAPi. In addition, the small inlet areas restrict the air flow into the RDE. The low air flows result in low average chamber pressures, which also are detrimental to EAPi. As the inlet area increases, the losses across the inlet decrease, the mass flow increases and the combustor chamber pressure increases.

The changes in RDE nozzle area have a similar effect on EAPi. As the nozzle area decreases, the combustion chamber pressure must increase to pass the same mass flow through the nozzle. This increases EAPi. Along with the mass flow effect, the reduced nozzle area back pressures the inlet by reducing the velocities in the inlet and thereby reducing the inlet's total pressure losses.

Overall, the results presented in Fig. 3 point toward the RDE design configurations most likely to demonstrate pressure gain. Those designs should have inlet areas of at least $40 \%$ of the combustor flow area and at least some amount of nozzle contraction (i.e. $A_{8} / A_{3.2}<1$ ).

While pressure gain does promise thermodynamic benefits, the reader is cautioned that a viable RDE needs to consider many factors and not just pressure gain. Additional factors require consideration such as insuring adequate thrust to meet vehicle demands, successful operation throughout the flight envelope, compatibility with upstream and downstream components, and adequate attention to fuel consumption. These additional demands may not always coincide with maximum pressure gain. Additionally, it should be kept in mind that these results assume no backflow. For real inlets, the effects of backflow may limit the practical extent of $A_{3.2} / A_{3.1}$.

There is one CFD solution point which does not trend well with the remaining results. That point is for the case with the air inlet minimum area ratio (0.6) equal to the nozzle minimum area ratio (0.6). For this point, the CFD does not achieve a converged solution because the mass fluxes and pressures continue to oscillate [18]. It is not known if this is a CFD numerical issue or an indication of some true RDE flow instabilities. Further study is required to resolve this phenomenon.

\section{E. Effect of Heat Release}

Effective heat release is a major function of any combustor. Therefore, the effect of heat release on the computed RDE EAPi was studied. For this study, the input heating value of the fuel was directly varied to affect the varied combustor heat release. Thus, to model $80 \%$ heat release, the heating value of the fuel used in the CFD was $80 \%$ of the maximum fuel heating value. By modeling in this fashion, the results reflect only global fuel heat release and does not address mixing or detonation/deflagration percentages. The resulting computed EAPi are shown in Fig. 4.

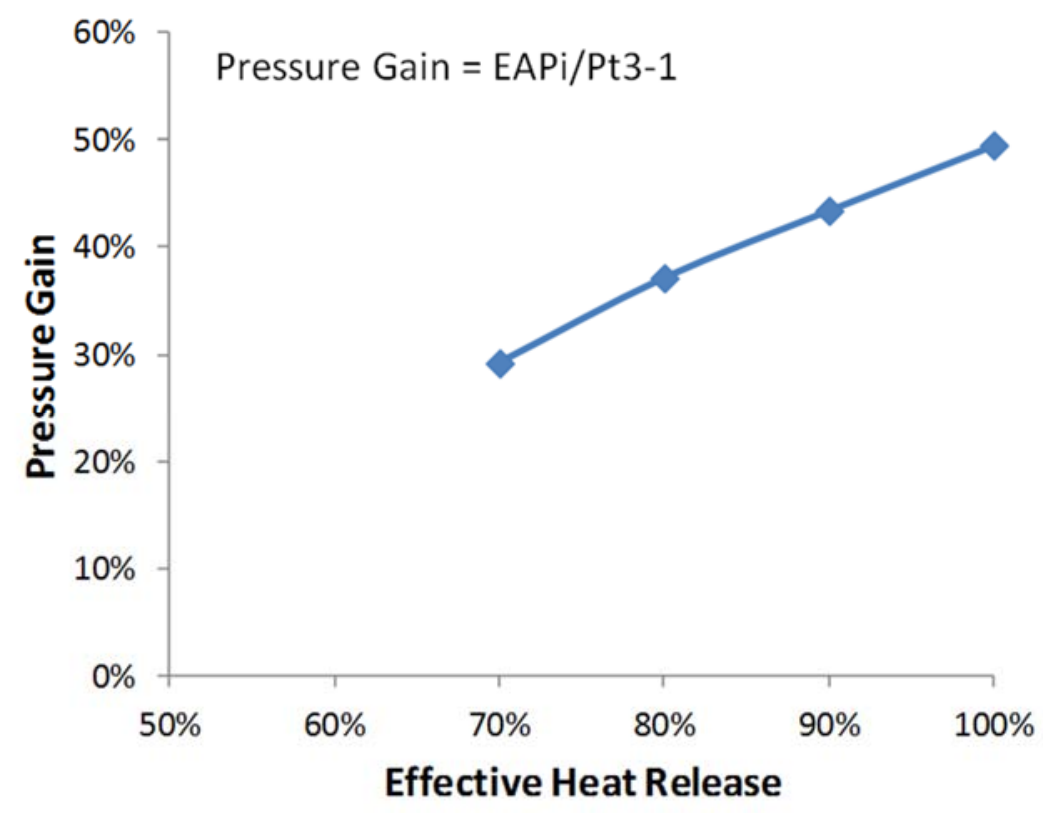

Fig. 4 Effect of Heat Release on Computed Combustor Pressure Gain 
The results of this study show a significant effect of the heat release on EAPi. Where there is less heat released, there is less volumetric flow through the nozzle, resulting in lower required chamber pressure and a similarly lower EAPi. Thus, EAPi will clearly attribute any issue with fuel heat release to the combustor.

\section{EAP Based on Experimental RDE Results}

Analysis of RDE CFD provides valuable insight into the fluid dynamics and thermodynamics of the device. However, the realization of PGC implementation still relies on experimental results to demonstrate engine performance. Therefore, a valid method for extracting a representative combustor total pressure from readily available experimental RDE results is desired.

To examine potential total pressure methodologies, the authors used the output of several RDE CFD solutions as surrogates for experimental data, assuming that the only available parameters were those available from experiments. Once again, following the rationale that EAP should represent available thrust or work, the EAP methodologies studied were required to be derived from the measured RDE thrust.

Several EAP methodologies were studied. The authors sought a methodology which was straightforward, could be easily implemented and would consistently provide results for EAP equal to or less than the analytical EAPi.

It is important to note that the authors sought a practical engineering solution to computing RDE EAP. The proposed method will provide, for most cases, representative results. However, to date, this proposed method has only been examined for RDE and therefore further study is required for additional forms of PGC. In addition, there are some known shortcomings of the method, explained below, that the experimenters must take into consideration.

\section{A. Isolating Combustor from Nozzle Performance}

Pressure gain combustion is a performance parameter associated with the combustor. But, representative combustor operation is dependent on having a representative back pressure. Therefore, to isolate the combustor performance from the nozzle performance, the combustor should be tested with the nozzle convergence but without the nozzle divergence. In addition, because the EAP value will be derived from the thrust of the combustor exhaust, special care must be taken when conducting the experiment to remove any forces that may find their way onto the thrust balance.

Figure 5 provides one approach to isolate the thrust of the RDE combustor. With the nozzle divergence removed, there remain significant base areas around the RDE combustor exit that will contribute axial forces to the thrust balance. Indeed, a previous simulation of an RDE with this geometry [19] indicated sub-ambient base pressures. Although the integrated base drag results were not presented, it was later found that the base drag was nearly $12 \%$ of the gross thrust. Failure to account for this drag when using the method to be described could reduce the EAP pressuregain to be described by as much as $38 \%$. Therefore, it is recommended that these base forces be subtracted from the balance readings by direct measurement of the static pressure forces acting on the base areas.

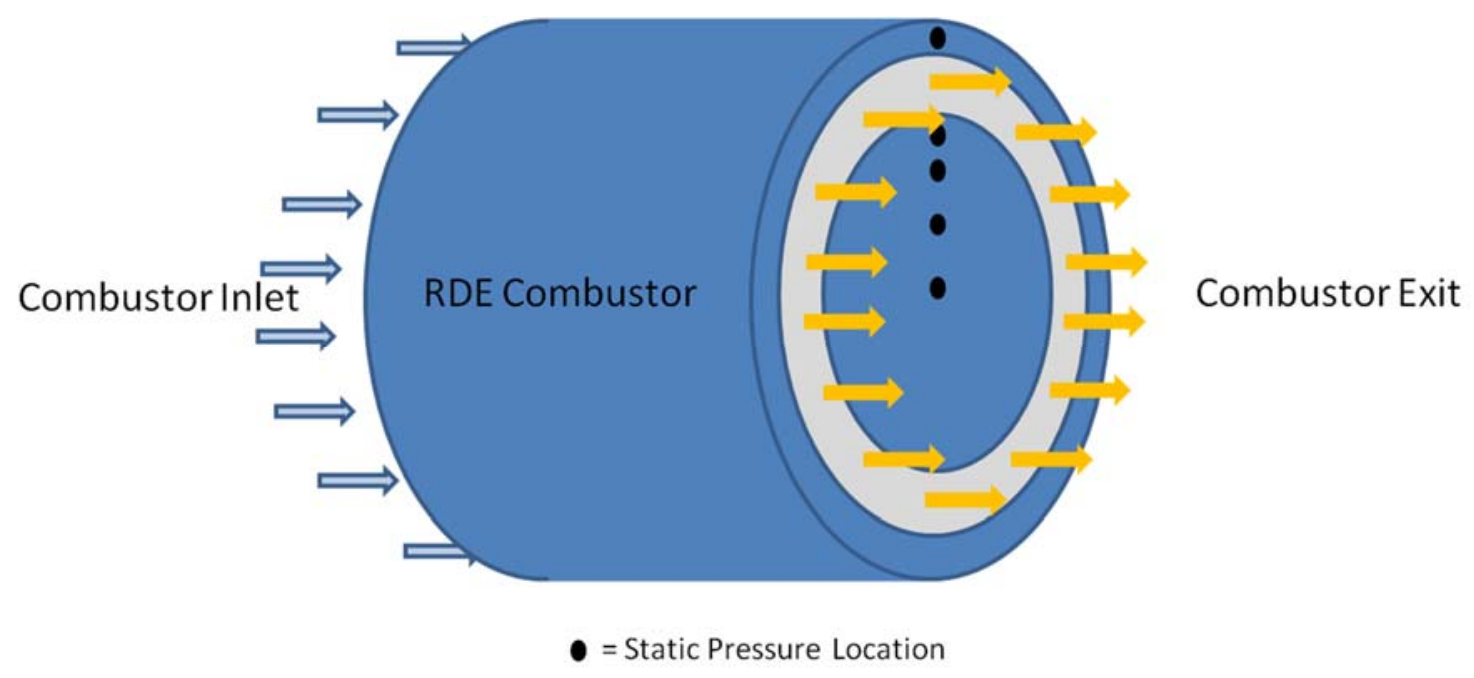

Fig. 5 Suggested RDE Combustor Test Setup 


\section{B. Proposed EAP Calculation Methodology from Experimental Data}

Once the true value of the combustor exit thrust is obtained, the estimate of the experimental EAP starts by examining the most basic form of the thrust equation:

$$
F_{g}=P_{8} A_{8}\left(1+\gamma M_{8 x}^{2}\right)-P_{0} A_{8}
$$

Rearranging for $P_{8}$ :

$$
\tilde{P}_{8}=\frac{\frac{F g}{A_{8}}+P_{0}}{1+\gamma M_{8 x}^{2}}
$$

As will be justified later in this paper, the axial exit Mach is assumed to be sonic:

$$
\tilde{P}_{8}=\frac{\frac{F g}{A_{8}}+P_{0}}{\gamma+1}
$$

The static pressure, $\tilde{P}_{8}$, will be used to compute an equivalent total pressure, $\tilde{P}_{t 8}$. This is done using the isentropic relationship for perfect gas:

$$
E A P=\tilde{P}_{t 8}=\tilde{P}_{8}\left(\frac{\gamma+1}{2}\right)^{\frac{\gamma}{\gamma-1}}
$$

\section{Justification for $\mathbf{M}_{8 \mathrm{x}}=1$ Assumption}

The non-uniform, unsteady nature of the RDE combustor exit flow creates challenges for equating this complex flow to a uniform steady flow. For most cases, the RDE combustor will have sufficient driving pressure to effectively choke the combustor exit. However, with a non-uniform flow, any choking condition (i.e. maximum mass flow rate) will have a range of axial Mach numbers as shown in Fig. 1. These Mach numbers typically will contain both subsonic and supersonic portions. Figure 6 provides the axial exit plane Mach numbers for the example CFD solution.

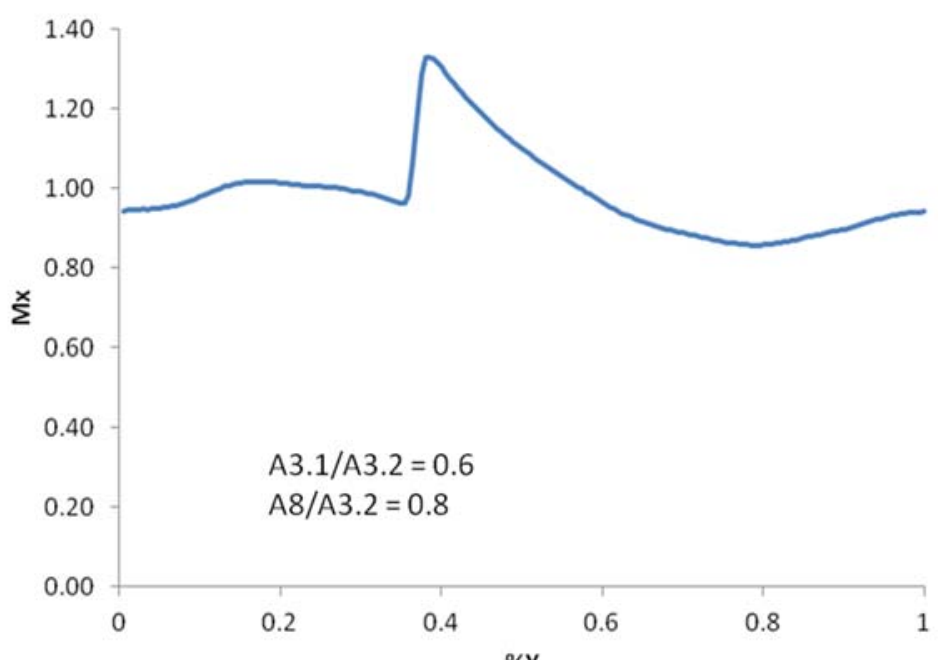

Fig. 6 Example of RDE Combustor Exit Axial Mach Number 
Because the majority of RDE CFD cases exhibit an exit that is effectively choked and have exit axial flow Mach numbers near 1 , a simple study was conducted to quantify the potentially induced error associated with assuming a uniform exit Mach number of 1 . To address this question, solutions for uniform flow, perfect gas cases assuming a constant $F_{g} / A$ were computed and the results shown in Fig. 7. The results show an assumption of Mach 1 at the exit results in the minimum possible computed EAP values. For the typical range of exit Mach numbers seen in RDE CFD solutions examined in this study, the assumption of Mach 1 introduces an EAP error of less than 5.4\%. Importantly, by assuming Mach 1, the EAP will be smaller than the EAP if any other Mach number were assumed. Thus, assuming a uniform exit Mach number of 1 provides an engineering estimated EAP that is conservative and is very close to EAPi.

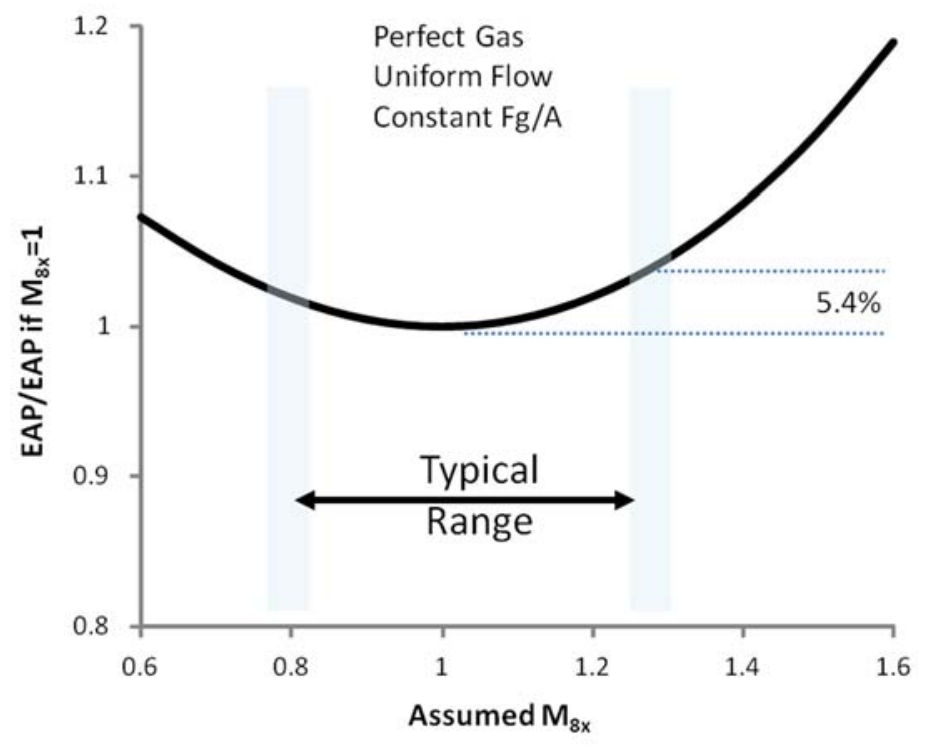

Fig. 7 Effect of Assumed Combustor Exit Axial Mach Number on Computed EAP

\section{Typical Results}

The CFD cases presented above in section V.D. were analyzed with the proposed EAP methodology. The results are presented in Fig. 8.

As the figure shows, the EAP computed from Fg show similar trends to the EAPi computed directly from the CFD results. Also, the EAP computed from Fg were consistently lower than the EAPi. This is due to two major factors: 1) as shown above, the Mach 1 assumption is conservative and 2) EAPi is based on ideal thrust while the EAP is based on actual thrust.

To help quantify the difference between EAPi and the thrust computed EAP, a correlation plot of the two parameters is presented in Fig. 9. Again, there is a good correlation between the two performance parameters, with the thrust computed EAP always less than the EAPi, but typically within $10 \%$.

\section{E. Effect of Heat Release}

The heat release study provided in section V.E. above was repeated for the EAP computed from gross thrust. The results shown in Fig. 10 exhibit a similar trend to the EAPi trend previously shown, although the EAP is consistently lower than the EAPi, for the reasons stated above.

\section{F. Non-Axial Energy Effect}

The simplified EAP methodology presented above in section VI.B. makes a subtle assumption regarding non-axial energy in that it assumes that the non-axial energy does not contribute to the measured axial thrust, $F_{g}$, and therefore will not contribute to the EAP. If the non-axial energy were included, then the total Mach number used to compute EAP from $P_{8}$ would be greater than 1 , resulting in a larger value of EAP. As an example, for the CFD case shown in Fig. 1, the inclusion of non-axial energy would result in a 3\% increase in computed EAP. 


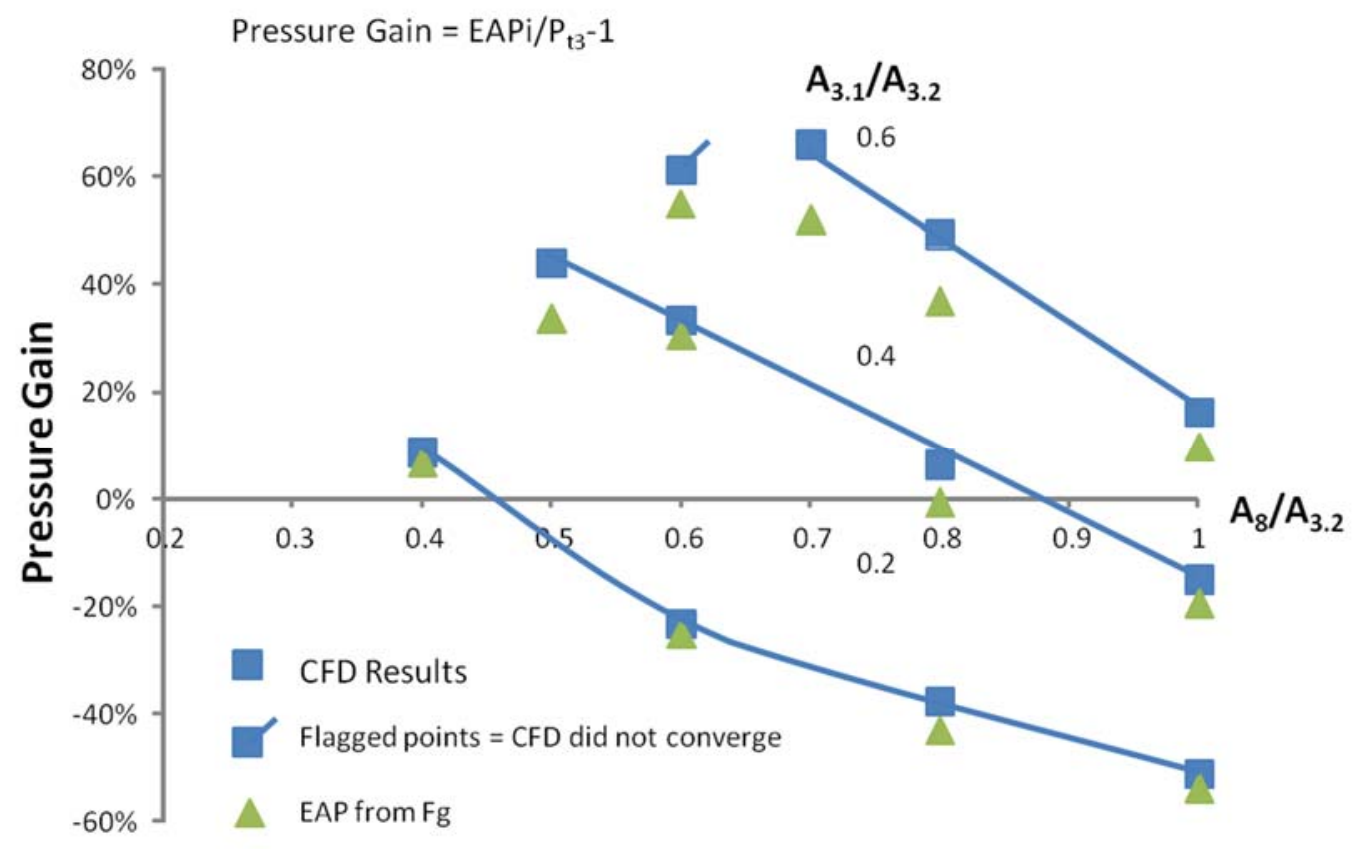

Fig. 8 Comparison of EAPi from CFD and EAP from $F_{g}$

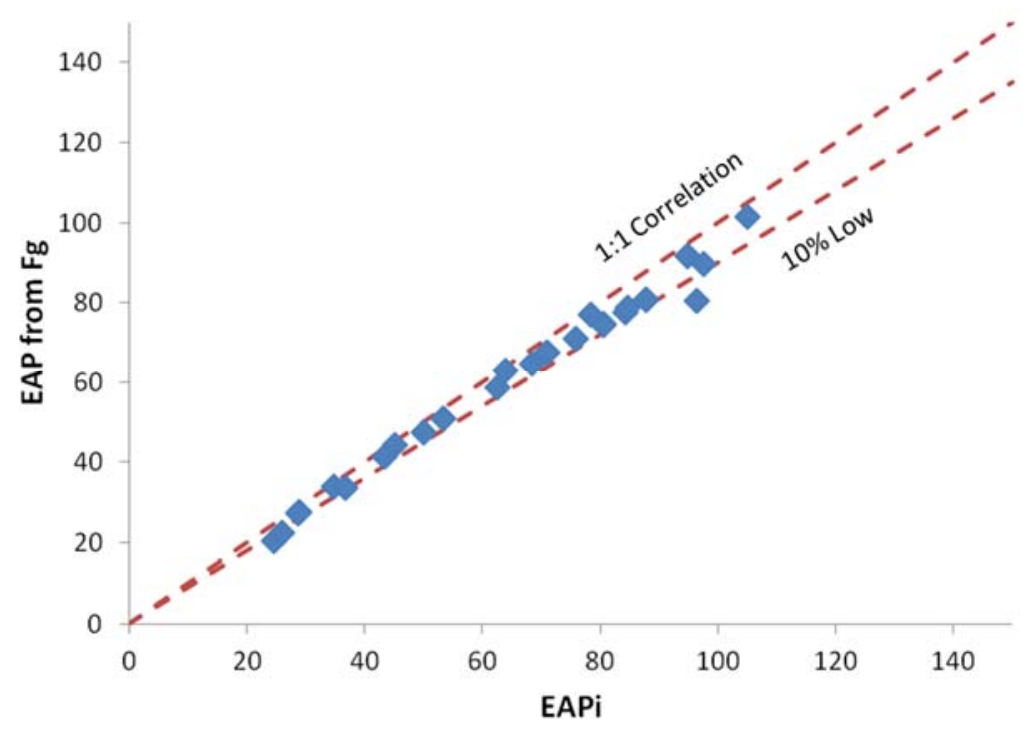

Fig. 9 Correlation of EAPi from CFD and EAP from $\mathrm{Fg}_{\mathrm{g}}$

\section{G. Comparison to Simple Averaging Techniques}

Researchers with complete RDE flow definition, such as CFD, may be tempted to use simple total pressure averaging techniques to define pressure gain. Similarly, experimenters may be tempted to use combustor static pressure instrumentation to infer pressure gain. However, as previously shown for the Atkinson (or Humphrey) cycle devices [2], simple averaging can produce significantly optimistic results. To illustrate the results of various averaging approaches, the example CFD case of Fig. 1 was post-processed using several calculation methods, with the results provided in Fig. 11. 


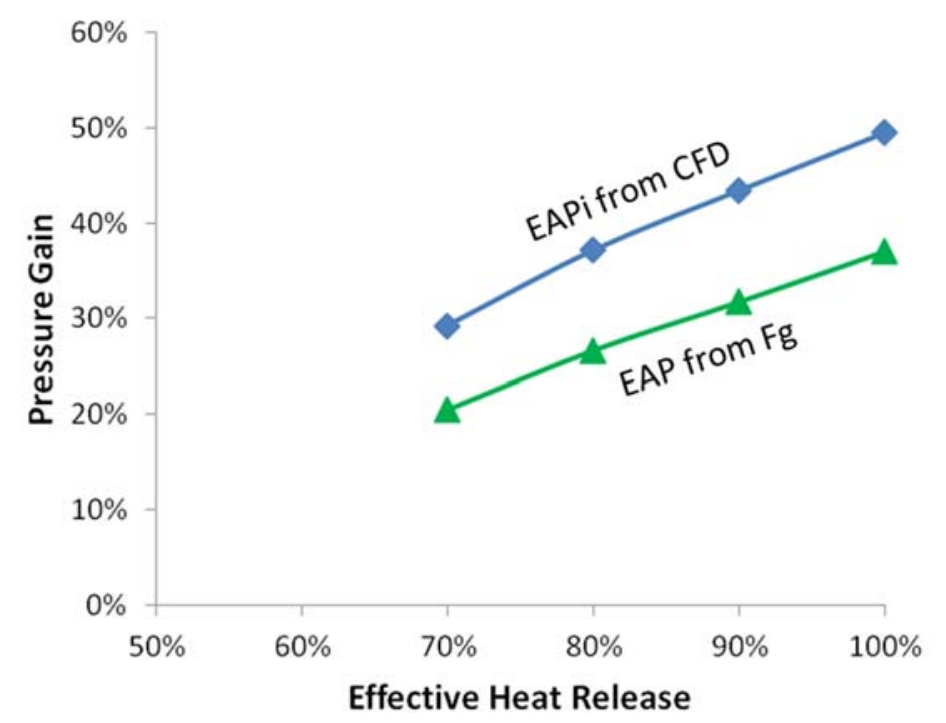

Fig. 10 Comparison of Heat Release Effect on Combustor Pressure Gain

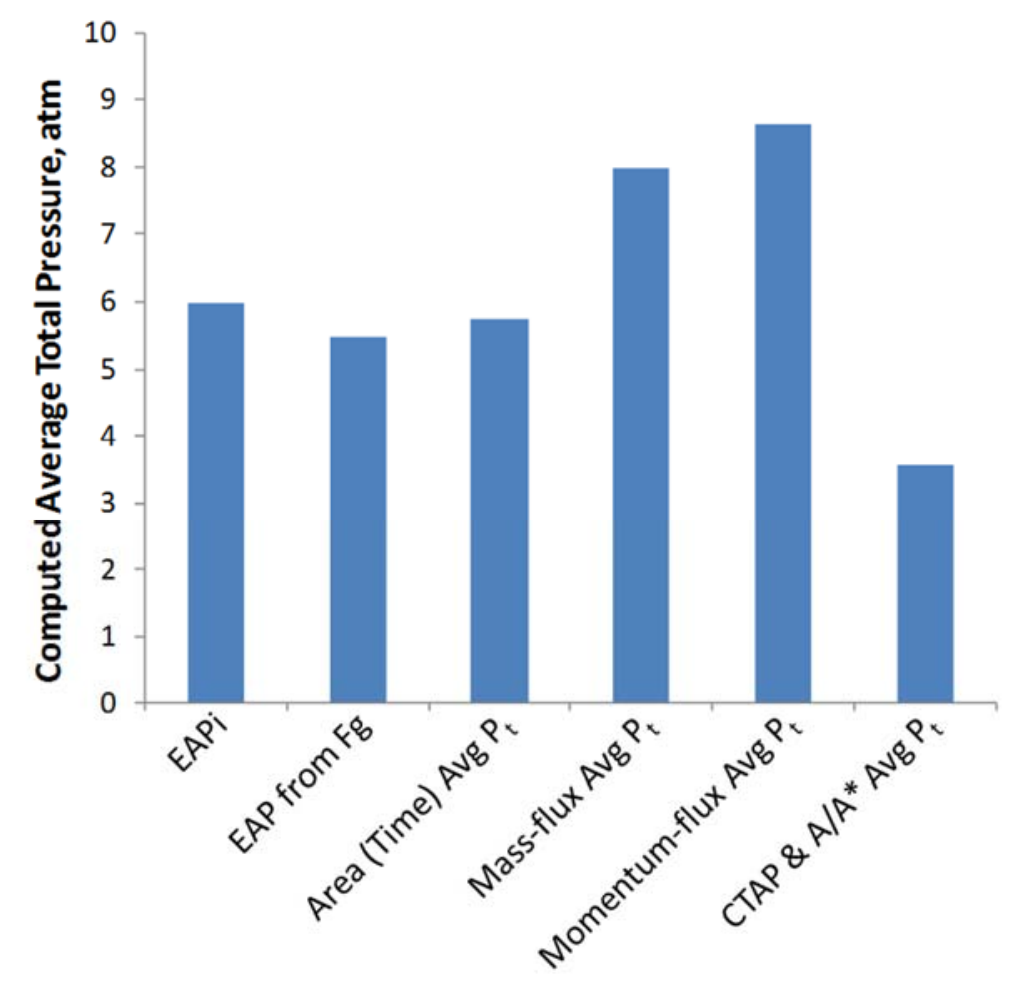

Figure 11 Comparison of Pressure Gain Calculation Methods

For this sample case, the proposed EAP from measured $F_{g}$ and the area (or time) averaged total pressure computed from CFD provided results similar to the computed EAPi. The CFD mass-flux averaged total pressure and the CFD momentum-flux average total pressure were significantly higher than EAPi. Finally, using the combustor exit average static pressure (analogous to an experimental CTAP measurement), and assuming $M_{8 x}=1$ with the physical $A_{3.2} / A_{8}$ to compute $M_{4}$, the resultant average total pressure is significantly lower than EAPi.

Based on this example, researchers are urged to use the EAPi method provided here to compute pressure gain from CFD or the EAP method to compute pressure gain from experimental measurements of RDE's. 


\section{H. User Cautions for Proposed EAP Methodology}

As stated previously, the proposed EAP methodology is an engineering solution to estimate RDE combustor pressure gain from measurable test data with reasonable accuracy, and almost uniform conservatism. For 25 of the 28 CFD cases studied, the computed EAP ranged from 1.7\% below EAPi to 8.7\% below EAPi. However, there were CFD cases where EAP was roughly 15\% below the EAPi. In these cases, the RDE combustors were operating with small overall pressure ratios (i.e. $P_{t 3} / P_{0}$ ) and/or high loss inlets. These configurations resulted in very low inlet exit total pressures $\left(\mathrm{P}_{t 3.2}\right)$, which in turn resulted in substantially subsonic exit Mach numbers (i.e. $M_{8 x} \sim 0.5$ ). This issue may make it difficult to demonstrate pressure gain for systems operating at low overall pressure ratios using this method.

\section{Conclusions}

A method has been proposed for demonstrating total pressure gain in a Pressure Gain Combustion (PGC) device. The method proposes using the Equivalent Available Pressure (EAP) to estimate the total pressure of a steady state combustor that would provide the equivalent thrust (or work) of the PGC device.

When a high fidelity flow simulation is available, a methodology for computing the ideal thrust (or ideal spouting velocity) from the CFD results is provided along with methodology to compute the ideal EAP (EAPi). The EAPi represents the total pressure of a steady, uniform flow with the same heat release that provides the same ideal thrust as the PGC device. The EAPi methodology should work for all unsteady devices.

In addition, an engineering estimation methodology for computing EAP from rotating detonation engine (RDE) testing measured thrust was provided. This estimation technique closely follows the EAPi for the same conditions, although the EAP is typically more conservative in estimating pressure gain than the EAPi. This EAP should provide a means toward actual demonstration of pressure gain in an RDE.

\section{Acknowledgments}

The authors would like to thank Dr. Doug Schwer of the Naval Research Laboratories for his assistance in preparing this paper.

\section{References}

[1] Paxson, D.E., Hoke, J.L., "Time Averaged Pressure Measurement in Fundamentally Unsteady Pressure Gain Combustion Systems," Proceedings JANNAF 45th Combustion Subcommittee, 33rd Airbreathing Propulsion Subcommittee, 27th Propulsion Systems Hazards Subcommittee, Monterey, CA, December, 2012, (also NASA/TM-2013-217826, January, 2013).

[2] Paxson, D.E., Kaemming, T.A., "Foundational Performance Analyses of Pressure Gain Combustion Thermodynamic Benefits for Gas Turbines,” AIAA 2012-0770, January 2012.

[3] Paxson, D. E., "Performance Evaluation Method for Ideal Airbreathing Pulse Detonation Engines," AIAA Journal of Propulsion and Power, Vol. 20, No. 5, 2004, pp. 945-947.

[4] Paxson, D.E., "Numerical Analysis of a Rotating Detonation Engine in the Relative Reference Frame," AIAA-2014-0284, Jan., 2014, (also NASA/TM 2014-216634, 2014).

[5] Paxson, D.E., Fotia, M.L., Hoke J.L., Schauer, F.R.“ Comparison of Numerically Simulated and Experimentally Measured Performance of a Rotating Detonation Engine,” AIAA-2015-1101, Jan., 2015.

[6] Rankin, B., Fotia, M. L., Paxson, D. E., Hoke, J. L., Schauer, F. R., "Experimental and Numerical Evaluation of Pressure Gain Combustion in a Rotating Detonation Engine," AIAA paper 2015-0877, January, 2015.

[7] Paxson, D.E., Naples, A. "Numerical and Analytical Assessment of a Coupled Rotating Detonation Engine and Turbine Experiment," AIAA-2017-1746, January, 2017.

[8] Theuerkauf, S. W., Schauer, F. R., Anthony, R. J., Paxson, D. E., Stevens, C. A. Hoke, J. L., "Comparison of Simulated and Measured Instantaneous Heat Flux in a Rotating Detonation Engine," AIAA 2016-1200, January, 2016.

[9] Paxson, D.E., Brophy, C.M. and Bruening, G.B., "Performance Evaluation of a Pulse Detonation Combustion Based Propulsion System Using Multiple Methods,” JANNAF Journal of Propulsion and Energetics, Vol. 3, No. 1, May, 2010, pp 44-54.

[10] Paxson, D.E., Fotia, M.L., Hoke J.L., Schauer, F.R.“ Comparison of Numerically Simulated and Experimentally Measured Performance of a Rotating Detonation Engine,” AIAA-2015-1101, Jan., 2015.

[11] Perkins, H.D., et. al., “An Assessment of Pulse Detonation Engine Performance Estimation Methods Based On Experimental Results,” AIAA 2005-3831, July, 2005.

[12] Paxson, D.E., Naples, A.G., Hoke, J.L., Schauer, F. "Numerical Analysis of a Pulse Detonation Cross Flow Heat Load Experiment,” AIAA-2011-584, January, 2011.

[13] Paxson, D.E., Schauer, F.R., Hopper, D., "Performance Impact of Deflagration to Detonation Transition Enhancing Obstacles,” AIAA-2009-0502, July, 2009, (also, NASA/TM-2012-217629). 
[14] Rankin, B., Fotia, M. L., Paxson, D. E., Hoke, J. L., Schauer, F. R., "Experimental and Numerical Evaluation of Pressure Gain Combustion in a Rotating Detonation Engine," AIAA paper 2015-0877, January, 2015.

[15] Paxson, D.E., “A General Numerical Model for Wave Rotor Analysis,” NASA TM 105740, 1992.

[16] Paxson, D.E., “An Improved Numerical Model for Wave Rotor Design and Analysis,” AIAA-93-0482, January, 1993.

[17] Rankin, B.A., Hoke, J. L., Schauer, F. R., " Periodic Exhaust Flow through a Converging-Diverging Nozzle Downstream of a Rotating Detonation Engine," AIAA paper 2014-1015, January, 2014.

[18] Paxson, D.E., "Impact of an Exhaust Throat on Semi-Idealized Rotating Detonation Engine Performance," AIAA paper 20161647, January, 2016.

[19] Schwer, D. A., Kailasanath, K., “Modeling Exhaust Effects in Rotating Detonation Engines,” AIAA 2012-3943, July, 2012. 